УДК 612.13.001.575

DOI: 10.17277/vestnik.2015.03.pp.424-428

\title{
MATHEMATICAL MODELING OF HEMODYNAMICS IN PATIENT-SPECIFIC MODEL OF CEREBRAL ANEURYSM*
}

\author{
S. V. Sindeev ${ }^{1}$, S. V. Frolov ${ }^{1}$, J. S. Bauer ${ }^{2}$ \\ Department "Biomedical Engineering”, TSTU (1); ssindeev@yandex.ru; \\ Neuroradiology Department of the Klinikum Rechts der Isar, \\ Technical University of Munich, Munich (Germany) (2)
}

Keywords: aneurysm; cardiovascular system; cerebral circulation; hemodynamics; mathematical model.

\begin{abstract}
Cerebral aneurysm is one of the major diseases of cerebral arteries. The hemodynamics plays a key role in aneurysm growth and rupture while an individual morphology of the cerebral artery is the most important factor which influences the aneurysm hemodynamics. The patient-specific model of aneurysm was taken for the evaluation of the blood flow into the aneurysm region. The numerical mesh was created for domain which consisted of 2 million elements. Using the developed software complex a numerical simulation was performed for the time period of $T=5 \mathrm{~s}$. According to the numerical results the unstable vortex exists in the aneurysm region which could lead to thrombus formation. The maximum velocity $-u=1,27 \mathrm{~m} / \mathrm{s}$ blood has at the inlet segment elbow while at the outlet segment a velocity does not exceed the $0,75 \mathrm{~m} / \mathrm{s}$. An intra-aneurysmal velocity magnitude varies from $4 \cdot 10^{-4}$ to $0,5 \mathrm{~m} / \mathrm{s}$. The lowest value of wall shear stress was obtained in the aneurysm dome, which can be a cause of the future growing and rupture of the studied aneurysm. The presented method can be used for the evaluation of the aneurysm flow-pattern for clinical decision support process.
\end{abstract}

Introduction. Cerebral aneurysm is one of the major diseases of cerebral arteries. According to the researchers [2-5] the hemodynamics plays a key role in aneurysm growth and rupture. Individual morphology of the cerebral artery is the most important factor which influences the aneurysm hemodynamics. The patient-specific evaluation of aneurysm hemodynamics is a promising method for investigation of intra-aneurysmal flow-pattern which helps the surgeon in decision making process on the pre-operational stage.

Methods. The patient-specific model of aneurysm was taken for the evaluation of the blood flow into the aneurysm region (Fig. 1). A selected cerebral region has one inlet and two outlets. The size of the cerebral segment is $35.8 \times 40 \times 79 \mathrm{~mm}$ with an aneurysm sac of $11.2 \times 11.4 \times 14.1 \mathrm{~mm}$. The inlet and outlet tubes have a diameter of $8 \mathrm{~mm}$.

* The reported study was supported by the Supercomputing Center of Lomonosov Moscow State University [1].

По материалам доклада на конференции «Актуальные проблемы энергосбережения и энергоэффективности в технических системах». 


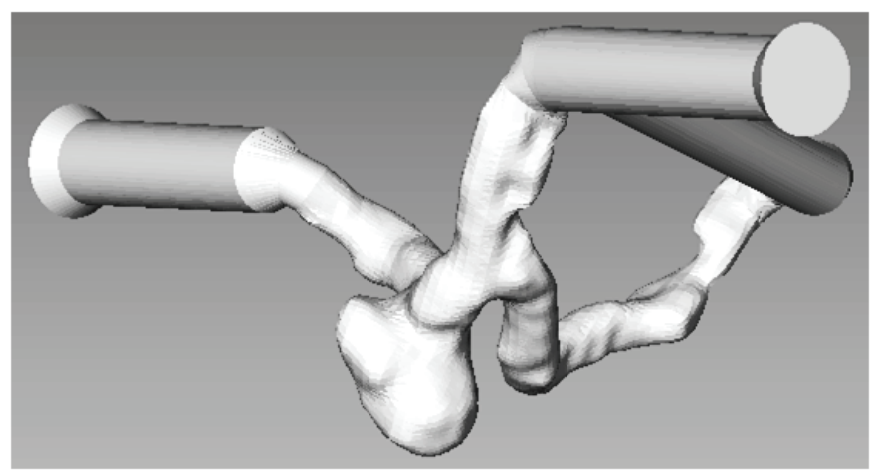

Fig. 1. Patient-specific geometry of the cerebral aneurysm

To describe the three-dimensional blood flow as incompressible Newtonian fluid we use the conservation momentum law:

$$
\frac{\partial u}{\partial t}+u \nabla u-v \Delta u+\frac{1}{\rho} \nabla P=f, \quad v=\frac{\mu}{\rho},
$$

where $u$ - blood velocity, $\mathrm{m} / \mathrm{s} ; v$ - kinematic blood viscosity, $\mathrm{m}^{2} / \mathrm{s} ; \mu$ - dynamic blood viscosity, $\mathrm{Pa} / \mathrm{s} ; \rho$ - blood density, $\mathrm{kg} / \mathrm{m}^{3} ; P$ - blood pressure, $\mathrm{Pa} ; f$ - external forces; $t$ - time, s.

Under external forces $f$ in equation (1) is usually understood gravity. This value in the simulation of hemodynamics, can be neglected $(f=0)$. Blood kinematic viscosity consider constant $v=$ const. Thus, in equation (1) there are two unknowns: blood velocity $u$ and pressure $P$ a continuity equation should be added:

$$
\frac{\partial \rho}{\partial t}+\operatorname{div} \rho u=0 .
$$

Since blood is modeled as incompressible Newtonian fluid, then $\rho=$ const. Therefore, equation (2) can be written as:

$$
\operatorname{div} u=0 .
$$

Thus, equations (1), (3) formed a closed equation system, describing blood flow through cerebral artery in three-dimensional space (3D hemodynamics model).

For solving system of equations (1) - (3) the proper initial and boundary conditions were applied. The no-slip condition was used for the vessel wall:

$$
\left.u\right|_{\text {wall }}=0 \text {. }
$$

For cerebral segment boundaries the boundary conditions can be applied by researcher. However most promising method is to obtain boundary conditions from a multiscale hemodynamics model [6]. In present case, the boundary conditions were taken from in vitro measurements from Interdisciplinary research library (IFL) of Klinikum Rechts der Isar. To apply the proper initial conditions $u_{0}=u(x, y, z, t)$ and $P_{0}=P(x, y, z, t)$ at $t=0$ the Stokes problem was solved:

$$
\left\{\begin{array}{l}
-v \Delta u_{0}+\nabla P_{0}=f \\
\text { div } u_{0}=0
\end{array}\right.
$$

In system (5) also as in the system (1), (3) influence of external forces neglected $(f=0)$. The boundary conditions are the same as for system (1) - (3).

Results. The numerical mesh was created for domain which consisted of 2 million elements. Using the developed software complex a numerical simulation was performed for the time period of $T=5 \mathrm{~s}$ with a time discretization period of $\Delta t=2 \cdot 10^{-4} \mathrm{~s}$. 


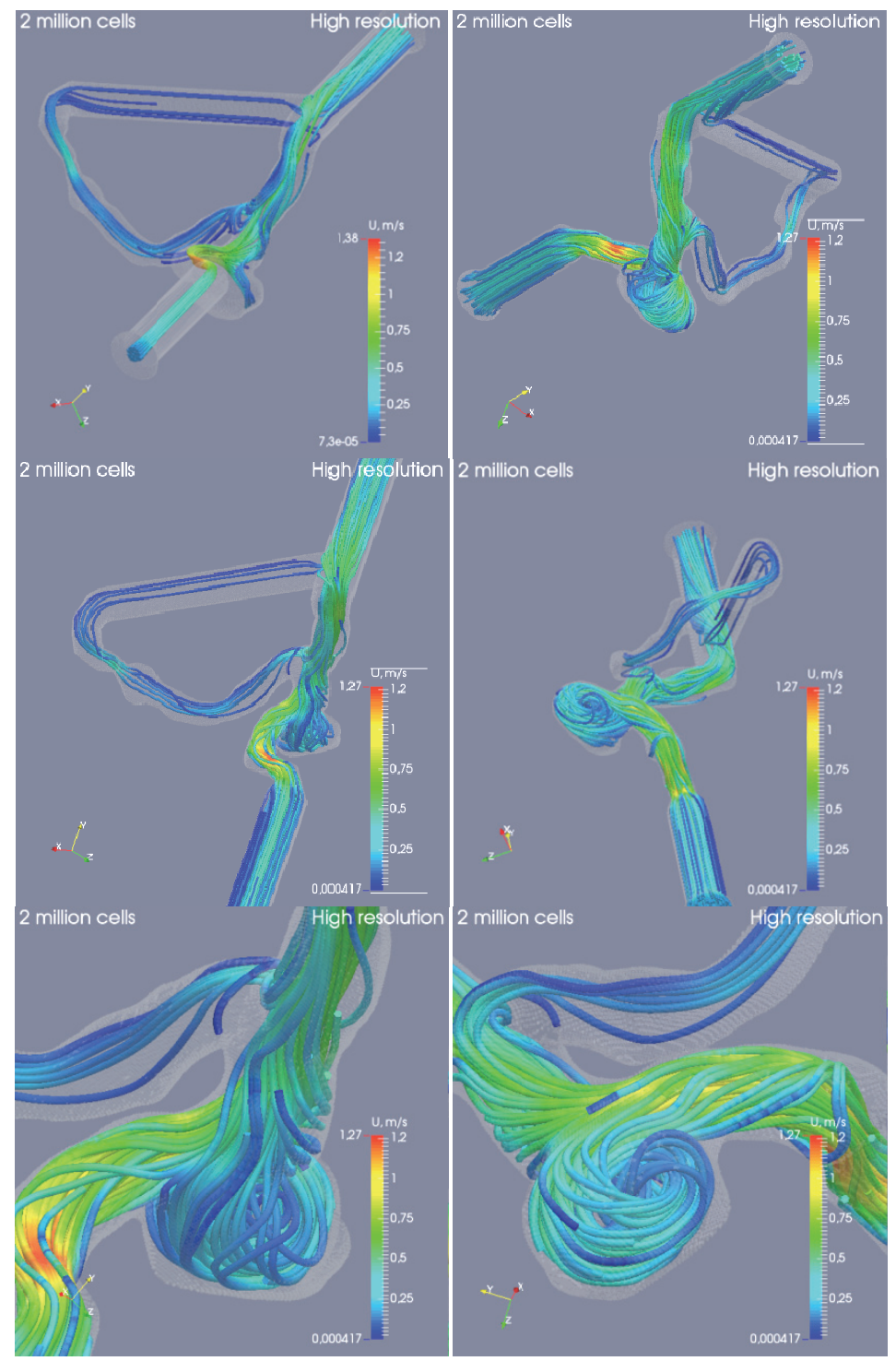

Fig. 2. Streamlines for the patient-specific aneurysm model of cerebral artery at systolic peak

The Lomonosov supercomputer was used for the numerical simulations. A duration of the cardiac cycle was $T_{\text {cardiac }}=1 \mathrm{~s}$ while the systolic period was $T_{\text {sys }}=0.2$. The streamlines for the systolic peak are shown in Fig. 2.

According to the numerical results the unstable vortex exists in the aneurysm region which could lead to thrombus formation. The maximum velocity $-u=1,27 \mathrm{~m} / \mathrm{s}$ blood has at the inlet segment elbow while at the outlet segment a velocity does not exceed the $0,75 \mathrm{~m} / \mathrm{s}$. An intra-aneurysmal velocity magnitude varies from $4 \cdot 10^{-4}$ to $0,5 \mathrm{~m} / \mathrm{s}$. The lowest value of wall shear stress was obtained in the aneurysm dome, which can be a cause of the future growing and rupture of the studied aneurysm.

Conclusion. The paper presents the quantitative analysis of the intra-aneurysmal hemodynamics in the patient-specific model of the cerebral aneurysm. The presented method can be used for the evaluation of the aneurysm flow-pattern for clinical decision support process. 


\title{
References
}

1. Voevodin Vl.V., Zhumatiy S.A., Sobolev S.I., Antonov A.S., Bryzgalov P.A., Nikitenko D.A., Stefanov K.S., Voevodin Vad.V. Open Systems J., 2012, no. 7, pp. 36-39.

2. Steiger H.J., Liepsch D.W., Poll A., Reulen H.J. Heart and Vessels, 1988, no. 4, pp. $162-169$.

3. Boussel L., Rayz V., McCulloch C., Martin C., Acevedo-Bolton G., Lawton M., Higashida R., Smith W., Young W., Saloner D. Stroke, 2008, vol. 39, no. 11, pp. 2997-3002.

4. Formaggia L., Quarteroni A., Veneziani A. Cardiovascular Mathematics. Modeling and simulation of cardiovascular system, Milan: Springer-Verlag, 2009, 522 p.

5. Valencia A., Guzmán A., Finol E., Amon C. Journal of Biomechanical Engineering, 2006, no. 4, pp. 516-526.

6. Frolov S.V., Sindeev S.V., Lischouk V.A., Gazizova D.Sh., Liepsch D., Balasso A. Boprosy sovremennoi nauki i praktiki. Universitet imeni V.I. Vernadskogo, 2013, no. 4(48), pp. 46-53.

\section{Математическое моделирование гемодинамики в индивидуализированной модели церебральной аневризмы}

\author{
С. В. Синдеев ${ }^{1}$, С. В. Фролов ${ }^{1}$, Я. Ш. Бауэр ${ }^{2}$
}

Кафедра «Биомедииинская техника», ФГБОУ ВПО «ТГТУ» (1); ssindeev@yandex.ru; нейрорадиологическое отделение клиники «Рехтс дер Изар», Технический университет Мюнхена, г. Мюнхен (Германия) (2)

Ключевые слова: аневризма; базилярная артерия; гемодинамика; математическая модель; сердечно-сосудистая система.

Аннотация: Использована индивидуализированная модель аневризмы для оценки кровотока в полости аневризмы. Построена вычислительная сетка, состоящая из двух миллионов элементов. С использованием разработанного программного обеспечения промоделирован период времени равный $T=5 \mathrm{c}$. Согласно численным результатам, в области аневризмы присутствует нестабильный вихрь, который может стать причиной тромбообразования. Максимальная скорость крови $u=1,27 \mathrm{~m} / \mathrm{c}$ наблюдается в изгибе входного сегмента, в то время как скорость крови в выходном сегменте не превосходит $0,75 \mathrm{~m} / \mathrm{c}$. Скорость крови в полости аневризмы изменяется от $4 \cdot 10^{-4}$ до $0,5 \mathrm{~m} / \mathrm{c}$. Наименьшее значение пристеночного напряжения сдвига обнаружено в куполе аневризмы, что может быть причиной дальнейшего роста и разрыва исследуемой аневризмы. Предложенный метод может быть использован для оценки кровотока в области аневризмы при поддержке принятия решений врача.

\section{Список литературы}

1. Практика суперкомпьютера «Ломоносов» / В. В. Воеводин [и др.] // Открытые системы. - 2012. - № 7. - Р. 36 - 39.

2. Hemodynamic Stress in Terminal Saccular Aneurysms: A laser-Doppler Study / H. J. Steiger [at al.] // Heart and Vessels. - 1988. - No. 4. - P. $162-169$.

3. Aneurysm Growth Occurs at Region of Low Wall Shear Stress: Patient-Specific Correlation of Hemodynamics and Growth in a Longitudinal Study / L. Boussel [at al.] // Stroke. - 2008. - Vol. 39, No. 11. - P. 2997 - 3002.

4. Formaggia, L. Cardiovascular Mathematics. Modeling and Simulation of Cardiovascular System / L. Formaggia, A. Quarteroni, A. Veneziani. - Milan : Springer-Verlag, 2009. $-522 \mathrm{p}$. 
5. Blood Flow Dynamics in Saccular Aneurysm Models of the Basilar Artery / A. Valencia [at al.] // Journal of Biomechanical Engineering. - 2006. - No. 4. - P. 516 - 526.

6. Development of Multiscale Hemodynamics Model for Research of Basilar Artery Circulation / S. V. Frolov [at al.] // Вопр. соврем. науки и практики. Ун-т им. В. И. Вернадского. - 2013. - № 4(48). - С. 46 - 53.

\section{Mathematische Modellierung der Hämodynamik im individualisierten Modell im Zerebralaneurysma}

Zusammenfassung: Das Zerebralaneurysma ist eines der wichtigen Erkrenkungen der Zrebralarterien. Die Schlüsselrole in dem Wachsen und in der Aneurysmaruptur spielt die Hämodynamik, die die individuele Morphologie der Zerebralarterie des Patientes wesentlich beeinflusst. In der Arbeit wurde das individuelle Modell des Aneurysmas für die Einschätzung der Blutströmung in der Aneurysmahöhle benutzt. Es wurde das Rechennetz, das aus 2 Millionen Elementen besteht, aufgebaut. Die entwickelte Software benutzend, wurde die Zeitperiode gleich $T=5$ Sekunden modelliert. Laut den zahlenmäßigen Ergebnissen gibt es auf dem Gebiet des Aneurysmas einen instabilen Wirbel, der als Grund der Blutpfropfbildung warden kann. Die maximale Geschwingigkeit des Blutes $u=1,27 \mathrm{~m} / \mathrm{s}$ wird in der Biegung des Eingangssegmentes beobachtet, während die Geschwindigkeit des Blutes im Ausgangssegment nicht größer als $0,75 \mathrm{~m} / \mathrm{s}$ ist. Die Geschwindigkeit des Blutes in der Aneurysmahöhle verändert sich von $4 \cdot 10^{-4}$ bis $0,5 \mathrm{~m} / \mathrm{s}$. Der kleinste Wert der Wandspannung der Verschiebung wurde in der Aneurysmakuppel aufgefunden, was einen Grund des weiteren Wachsen und der Ruptur des untersuchenden Aneurysmas sein kann. Die vorgeschlagene Methode kann für die Einschätzung der Blutströmung auf dem Gebiet des Aneurysmas bei der Unterhaltung des Arztbeschlußfassen benutzt sein.

\section{Modélage mathématique de l'hémodynamique dans un modèle individualisé de l'anévrisme cérébral}

Résumé: L'anévrisme cérébral est l'une des principales maladies des artères cérébrales. Le rôle clé dans la croissance et dans la rupture de l'anévrisme joue l'hémodynamique, qui est significativement affectée par la morphologie individuelle de l' artère cérébrale d'un patient. Dans l'article a été utilisé le modèle individualisé de l'anévrisme pour évaluer le flux sanguin dans la cavité de l'anévrisme. A été construite la grille de calcul composée de 2 millions d'éléments. En utilisant un logiciel développé, a été modélée une période de temps égale à $T=5$ secondes. Selon les résultats numériques, dans le domaine de l'anévrisme est présent un tourbillon instable qui peut devenir la cause de la formation de thrombus. La vitesse maximale de sang $u=1,27 \mathrm{~m} / \mathrm{s}$ est observée dans un méandre de l'entrée d'un segment, alors que la vitesse du sang dans le segment de sortie ne dépasse pas de $0,75 \mathrm{~m} / \mathrm{s}$. La vitesse du sang dans la cavité d'un anévrisme change de $4 \cdot 10^{-4}$ à $0,5 \mathrm{~m} / \mathrm{s}$. La plus petite valeur de la tension du décallage a été détectée dans la coupole de l'anévrisme, ce qui peut être la cause de la poursuite de la croissance et de la rupture de l'anévrisme étudié. La méthode proposée peut être utilisé pour évaluer le flux sanguin dans le domaine de l'anévrisme avec l'appui de la prise de la décision par le médecin.

Авторы: Синдеев Сергей Вячеславович - аспирант кафедры «Биомедицинская техника»; Фролов Сергей Владимирович - доктор технических наук, професcop, заведующий кафедрой «Биомедицинская техника», ФГБОУ ВПО «ТГТУ»; Бауэр Яи Штефан - PhD, профессор, врач нейрорадиологического отделения клиники «Рехтс дер Изар», Технический университет Мюнхена, г. Мюнхен (Германия).

Рецензент: Гатапова Наталья Цибиковна - доктор технических наук, профессор, заведующая кафедрой «Технологические процессы, аппараты и техносферная безопасность», ФГБОУ ВПО «ТГТУ». 